\title{
Uma Revisão Sistemática sobre Interações de Halogênio em Derivados de Nitrobenzeno Substituídos
}

\author{
Ricardo R. Ternavisk, Gerardo A. Hernández, Jean M. F. Custódio, Ademir J. \\ Camargo \& Hamilton B. Napolitano
}

Este trabalho buscou analisar as interações de halogênio observadas em compostos derivados de 2,6-dibromonitrobenzeno, encontradas na literatura. As pesquisas foram realizadas nas bases de dados: Google Academic, Springer, PubMed e Science Direct. Foram identificados 102 trabalhos na busca inicial, dos quais somente 11 foram selecionados depois da aplicação dos critérios de inclusão e exclusão e da leitura dos trabalhos. Baseado nos trabalhos selecionados, somente parâmetros geométricos são considerados para investigação das interações de halogênio, cerca de 7 trabalhos. A partir desses dados, infere-se a demanda por uma análise mais detalhada do ponto de vista teórico das interações envolvendo átomos de halogênio para essa classe de compostos. Sobretudo, considerando as interações entre dois átomos da mesma família.

Palavras chave: interações de halogênio; DFT; nitrobenzeno.

This work aimed to analyze the halogen interactions observed in the 2,6-dibromonitrobenzene derivatives found in the literature. The searches were conducted in databases: Google Academic, Springer, PubMed and Science Direct. We identified 102 papers in the initial search of which only 11 were selected after the application of the inclusion and exclusion criteria and the reading of the papers. Based on the selected papers, only geometric parameters are considered for the investigation of halogen interactions, about 7 papers. From these data, we infer the demand for a more detailed analysis from the theoretical point of view of the interactions involving halogen atoms for this class of compounds. Especially considering the interactions between two atoms of the same species.

Keywords: Halogen bonding; DFT; nitrobenzene. 


\section{Introdução}

Uma ligação de halogênio é uma interação não covalente entre uma região eletrofilica associada a um átomo de halogênio de uma molécula e uma região nucleofílica em outra molécula. Nesse caso, o halogênio age como eletrófilo, apesar de sua alta eletronegatividade. Esse tipo de ligação de halogênio tem atraído grande atenção devido à sua importância na química supramolecular e à engenharia de materiais. Essa classe de interações desempenha papéis importantes na bioquímica, como complexos proteína-ligante, além de muitas aplicações em química e química de materiais e na concepção de novos materiais em uma variedade de áreas como fotônica, óticas não-lineares e farmacêutica ${ }^{1,2}$.

Interações de halogênio são altamente direcionais de curto alcance $\mathrm{R} 1-\mathrm{X} \cdot \cdots \mathrm{Y}-\mathrm{R} 2$, na qual $\mathrm{X}$ é um halogênio (cloro, bromo ou iodo), Y geralmente é um nucleófilo com pelo menos um par de elétrons livres (oxigênio, nitrogênio ou enxofre ou ainda uma carbonila ou hidroxila) e R são radicais. As primeiras evidências das interações de halogênio datam de antes de 1814 quando M. Colin estudou o comportamento do iodo reagindo com amônia e reportou a formação de um di-iodo/amônia. Em 1863, F Guthries purificou sistema e o formulou como $\mathrm{NH}_{3} \mathrm{I}_{2}$, adicionando $\mathrm{I}_{2}$ a uma solução saturada de nitrato de amônio, que se decompunha quando era exposto ao ar em amônia e $I_{2}$. Interações envolvendo o bromo e o cloro foram obtidas logo após o uso de complexos orgânicos como doadores e aceitadores de interações de halogênios. Tais interações foram relatadas pela primeira vez em 1883 por O. Roussopoulos que descreveu a interação entre iodofórmio e a quinolina entre outras estruturas ${ }^{1}$.

Por volta de 1949, Benesi e Hildebrand ${ }^{3,4}$ nomearam incialmente essas interações como complexos de transferência de cargas. Em 1950, Mulliken ${ }^{5}$ renomeou como interações doador-aceitador. Essas interações foram atribuídas à transferência de cargas entre o oxigênio, nitrogênio ou o enxofre (bases de Lewis) e um átomo de halogênio polarizado (ácido de Lewis).

Os estudos cristalográficos de Hassel e colaboradores em $1959^{6}$ mostraram que os produtos de reação entre bromo e 1,4-dioxano apresentavam curtas distâncias intermoleculares entre os átomos de $\mathrm{O}$ e $\mathrm{Br}(2,71 \AA)$, e ângulos de ligação $\mathrm{C}-\mathrm{Br} \cdot \cdots \mathrm{O}$ próximos de $180^{\circ}$. Hassel foi laureada com o prêmio Nobel em $1969^{7}$ por suas pesquisas que mostravam que os halogênio podem atuar como aceitador de elétrons, formando complexos de transferência de cargas altamente direcionais. Hassel e colaboradores, em $1970^{8}$, estudaram os aspectos estruturais relacionados a essa classe de interações em estruturas cristalinas, utilizando o termo ligação de halogênio para se referir a tais interações e reportando ainda a forte analogia entre essas interações e as interações clássicas de hidrogênio ${ }^{1,2}$.

Em 2007, Politzer e colaboradores ${ }^{9}$ introduziram o conceito $\sigma$-hole para explicar a existência das interações de halogênio. Em alguns sistemas, a polarização das cargas se concentra ao longo da interação, o que por sua vez acaba induzindo uma deficiência eletrônica do lado oposto, denominada $\sigma$-hole ${ }^{1,10,9}$. A Figura 1 ilustra a região do $\sigma$-hole9 na molécula de 1,3-dibromo-2-X-5-nitrobenzeno, no qual X são os elementos $\mathrm{F}, \mathrm{Cl}, \mathrm{Br}$ e I respectivamente. Observa-se a região positiva do $\sigma$-hole ${ }^{9}$, no qual os máximos de superfície correspondem à energia de $21,2 \mathrm{Kcal} / \mathrm{mol}$, $18,8 \mathrm{Kcal} / \mathrm{mol}$ e $12,7 \mathrm{Kcal} / \mathrm{mol}$ para o iodo, bromo e cloro respectivamente e uma região parcialmente negativa sobre o átomo de flúor com máximo de energia em $-4,6 \mathrm{Kcal} / \mathrm{mol}$. Para contatos com nucleófilos, como oxigênio e nitrogênio, os ângulos observados estão entre $160^{\circ}$ e $180^{\circ}$. Entretanto, essa tendência não é observada para átomos de flúor. Existem ainda os contatos de halogênio $\mathrm{X} \bullet \boldsymbol{X}^{\mathrm{X}}$ que são mais difíceis de conceituar porque a transferência de cargas nesse tipo de contato $(\mathrm{Br} \bullet \bullet B r$ e I $\bullet \bullet B r)$ não é tão óbvia ${ }^{1}$.

Compostos derivados de 1,3-Dibromo-nitrobenzeno, Figura 2, são promissores pois possuem o grupo nitro ligado ao anel benzeno que atua como uma base de Lewis forte. Devido à alta densidade eletrônica dos oxigênios e ao seu efeito indutivo, retirador de elétrons, juntamente aos halogênios na posição para em relação ao grupo nitro, podem favorecer o surgimento de interações de halogênio. Além da formação de um complexo de transferência de cargas que colabora para o surgimento de propriedades óticas não lineares. 


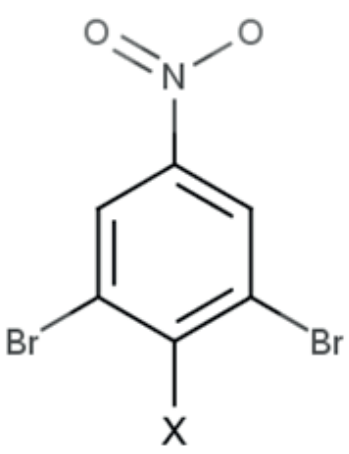

$\mathrm{X}=(\mathrm{F}, \mathrm{Cl}, \mathrm{Br}, \mathrm{I})$

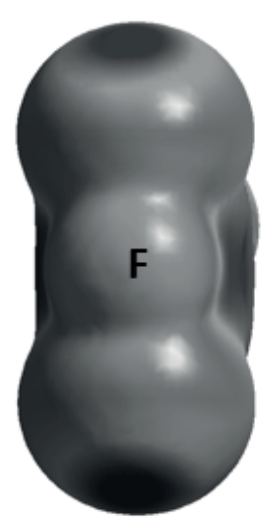

$-25 \mathrm{Kcal} / \mathrm{mol}$
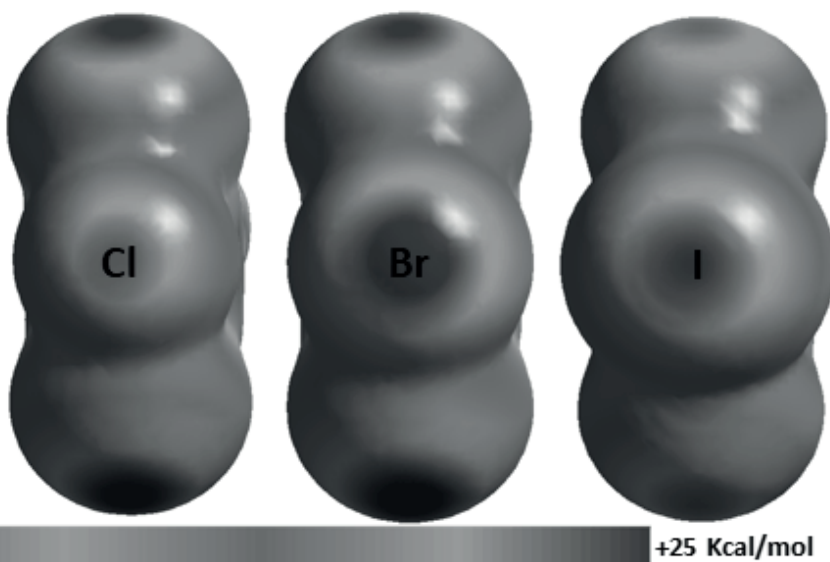

Figura 1. Superfície de potencial eletrostático da molécula 1,3-dibromo-2-X-5nitrobenzeno, calculado com nível B3LYP/6-31G(d,p) e isodensidade de 0,0004. Regiões representadas em azul possuem a nuvem eletrônica mais deslocada e cargas parciais positivas e, em vermelho, as regiões com cargas parciais negativas que possuem alta densidade eletrônica.

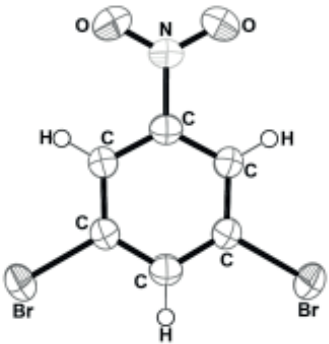

(1)

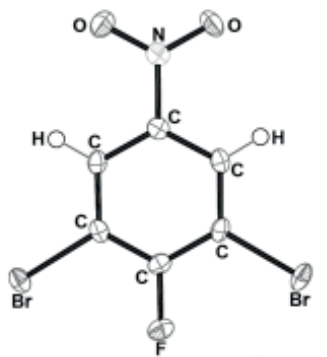

(2)

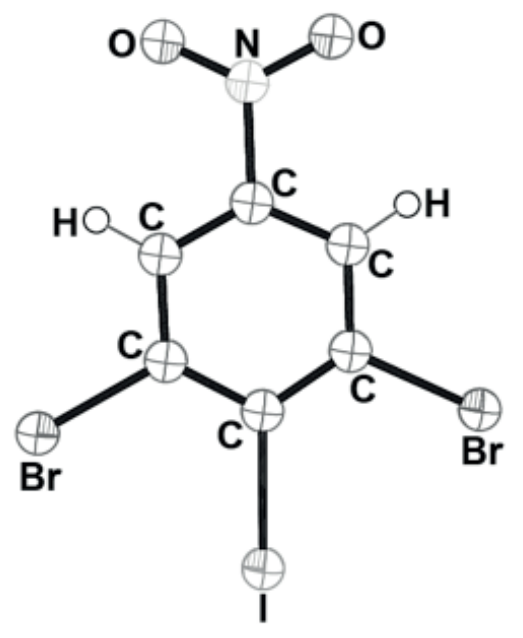

(3)

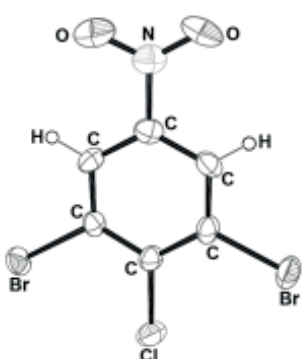

(4)

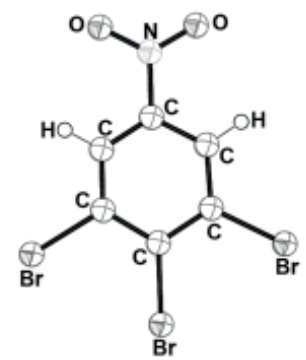

(5)

Figura 2. Representação Ortep, com elipsoides a 50\% dos compostos 1,3-Dibromo-5-nitrobenzeno (1), 1,3-Dibromo-2-fluor-5-nitrobenzeno (2), 1,3-Dibromo-2-iodo-5-nitrobenzeno (3), 1,3-Dibromo-2-cloro-5-nitrobenzeno (4) e 1,2,3-Tribromo-5-nitrobenzeno ${ }^{5}$. 
Além dos parâmetros geométricos, metodologias teóricas podem ser empregadas com o intuito de analisar a influência dessas interações no empacotamento cristalino. Considerando que muitos dados não podem ser obtidos diretamente de experimentos em estado sólido, cálculos teóricos como mapa de potencial eletrostático, superfície de Hirshfeld ${ }_{11}$, energia de interação, QTAIM ${ }_{12}$ (Quantum Theory of Atoms in Molecules) e orbitais naturais de ligação (NBOs) $)^{13,14,15}$ são ferramentas poderosas para explicar a formação dessas interações, bem como seus impactos no empacotamento molecular. Dentre os métodos mais utilizados no estudo de interações moleculares, podemos citar os métodos perturbativos como a teoria de perturbação de Moller-Pleset, Coupled Cluster, Teoria do Funcional de Densidade $(\mathrm{DFT})^{16}$ e semiempíricos ${ }^{1,10}$.

\section{Metodologia}

Foram utilizadas as bases de dados: Google Scholar, Springer, PubMed e Science Direct. A pesquisa foi realizada com a seguintes strings: nitrobenzene, AND halogen AND bond, AND crystallography AND "halogen bond" AND (crystal) AND NOT (protein, AND metal). Não foi adicionada restrição quanto ao período, restando, assim, somente as restrições das respectivas plataformas.

Todos os artigos encontrados foram identificados e posteriormente selecionados de acordo com o objetivo da pesquisa, a partir dos títulos e resumos. Nesse sentido, trabalhos que incluíam interações de halogênios com metais ou sítios biológicos não foram considerados nessa revisão. Os artigos selecionados, nessa primeira etapa, foram novamente avaliados a partir da leitura na íntegra e classificados quanto à sua relevância para o estudo em questão. Artigos que apresentavam somente a descrição geométrica das interações foram classificados como de baixa prioridade enquanto artigos que apresentavam análise experimental e teórica das interações como de alta prioridade, todos possuindo o núcleo nitrobenzeno. As estruturas dos trabalhos deveriam conter, pelo menos, o núcleo nitrobenzeno. Foram avaliadas tanto a metodologia utilizada para analisar as interações intermoleculares de halogênios bem como o nível de cálculo empregados.
Após a leitura dos artigos, uma nova busca com pequenas alterações nas strings foi realizada, objetivando verificar possíveis trabalhos que não haviam sido identificados nas buscas iniciais. Foram identificados mais 2 trabalhos, selecionados para segunda etapa, devido à relevância de seus resultados, bem como da metodologia empregada.

\section{Resultados e Discussões}

Após a aplicação dos filtros de busca e dos critérios de inclusão e exclusão, 11 trabalhos foram utilizados para a revisão sistemática. Gavezotti ${ }^{17}$ analisou as interações intermoleculares entre átomos de halogênio $(\mathrm{Cl}, \mathrm{Br}$, e I) e $\mathrm{O}$ ou $\mathrm{N}$, em cristais orgânicos de moléculas, depositadas no $\mathrm{CCDC}^{18}$, abrangendo cerca de 10.938 estruturas cristalinas. As moléculas selecionadas deveriam ter menos de 50 átomos, não apresentarem problemas de desordem, não possuírem mais do que duas moléculas por unidade assimétrica e um R-factor $<0,07517$. Gavezotti utilizou o software GAUSSIAN para calcular a densidade de carga, utilizando o nível de cálculo MP2/6-31G**. Posteriormente, utilizou-se o software PIXEL para calcular a Distance density function (DDF). Essa ferramenta é conveniente para o estudo dos dados geométricos (distância e ângulo de ligação) em coleções de estruturas cristalinas. A Tabela 1 resume os parâmetros geométricos encontrados para estruturas cristalinas do trabalho de Gavezotti ${ }^{17}$.

Embora Gavezotti tenha feito uso da metodologia

Tabela 1. Resultados obtidos por Gavezotti. Levantamento de dados realizado no CCDC. Distância entre os átomos X $\cdots Y$ Y.

\begin{tabular}{|c|c|c|c|}
\hline Contatos & $\begin{array}{c}\mathbf{N}^{\mathbf{0}} \text { de } \\
\text { estruturas }\end{array}$ & $\begin{array}{c}\text { Raio intermo- } \\
\text { lecular }(\AA)\end{array}$ & $\begin{array}{c}\text { Menor } \\
\text { distância ob- } \\
\text { servada }(\AA)\end{array}$ \\
\hline $\mathrm{Cl} \ldots \mathrm{N}$ & 2954 & 3,34 & 2,95 \\
\hline $\mathrm{Br} . . \mathrm{N}$ & 1318 & 3,51 & 2,72 \\
\hline $\mathrm{I} \ldots \mathrm{O}$ & 417 & 3,67 & 2,75 \\
\hline $\mathrm{Cl} \ldots \mathrm{O}$ & 3699 & 3,40 & 2,79 \\
\hline $\mathrm{Br} . . \mathrm{O}$ & 2007 & 3,45 & 2,85 \\
\hline $\mathrm{I} \ldots \mathrm{O}$ & 547 & 3,61 & 2,76 \\
\hline
\end{tabular}


teórica, sua abordagem ainda é fortemente baseada na geometria das interações e ele não realizou estudos teóricos mais profundos ,a fim de estudar os mecanismos envolvidos nessas interações. Entretanto, os dados levantados em seu artigo são de extrema relevância, considerando que uma grande quantidade de estruturas foi estudada e seus parâmetros geométricos descritos rigorosamente.

Klaus $\mathrm{Merz}^{20}$ analisou a influência do iodo no empacotamento cristalino de benzonitrilos e fenóis ,substituídos a partir da determinação estrutural de 3 novos compostos: m-iodobenzonitrilo, m-iodofenol e p-iodobenzonitrilo. As interações foram comparadas a informações geométricas em moléculas derivadas de nitrobenzeno substituídas. Klaus analisou as cargas derivadas das análise populacional de Mulliken, obtidas por métodos ab intio (DFT $)^{16}$ o que auxiliou na determinação do aceitador e do doador nas interações. Foram observadas interações do tipo $\mathrm{I} \bullet \bullet \mathrm{I}, \mathrm{I} \bullet \bullet \mathrm{O}, \mathrm{C}-\mathrm{N} \bullet \bullet \mathrm{I}$ com distâncias de $3,332,3,919$ e 4,080 $\AA$ para interações I $\bullet \bullet O$ do iodophenol meta, orto e para substituídos, repectivamente ${ }^{20}$.

Izabella Mossakowska e Grazina M. Wójcik ${ }^{21}$, estudaram as interações intermoleculares no empacotamento molecular experimental e teórico de 30 cristais de nitrobenzenos monossubstituídos. As interações que envolviam átomos de halogênio em halogenonitrobenzenos foram identificadas como $\mathrm{Cl} \bullet \cdot \mathrm{O}, \mathrm{Br} \bullet \cdot \mathrm{O}$ e uma interação bifurcada $\mathrm{I} \bullet \bullet \mathrm{O}$ nas estruturas orto-cloronitrobenzeno, meta-bromonitrobenzeno e para-iodonitrobenzeno, respectivamente. As interações foram observadas utilizando os padrões do Mercury 3.1 para interações intermoleculares, ou seja, utilizou-se somente parâmetros geométricos para avaliação das interações ${ }^{21}$.

Mohammad Mazharol Hoque e colaboradores ${ }^{22}$ relataram um método para iodização direta de anilinas substituídas como iodo molecular e acetato de cobre em ácido acético produzindo 2-iodoacetanilias e 2-iodoanilinas. Embora as estruturas foram elucidadas por espectroscopia no infravermelho e não por difração de raios-X, o artigo foi mantido como selecionado tendo em vista a metodologia teórica aplicada para estudo de possíveis interações com halogênio ${ }^{22}$. Foi utilizado o nível de cálculo B3LYP/MidiX para os cálculos teóricos. Após a otimização da geometria, foram calculadas as cargas de Mulliken, os orbitais (HOMO, Highest Occupied Molecular Ortial) orbital molecular mais alto ocupado e (LUMO, Lowest Unoccupied Molecular Orbital) orbital molecular não ocupado mais baixo, bem como energias de ligação e propriedades termodinâmicas como entalpia e energia livre de Gibbs. O mapa de potencial eletrostático deixa evidente a formação da região $\sigma$-hole ${ }^{9}$ nos átomos de iodo. Os cálculos teóricos confirmam que esses compostos iodados podem facilmente participar de ligações de halogênio. Entretanto, não há menção do motivo de escolha de tal nível de teoria, e nem a comparação com outros métodos ${ }^{22}$.

José A. Romero e colaboradores publicaram, em 2015, as estruturas do 1,2,3-tribromo-5-nitrobenzeno e 1,3-dibromo-2-iodo-5-nitrobenzeno ${ }^{23}$. Eles classificaram as interações segundo um esquema baseado em padrões geométricos desenvolvido por Desiraju. As Tabelas 2 e 3 indicam os padrões geométricos encontrados para interações de halogênio nos dois compostos estudados por Romero $^{23}$.

Tabela 2. Geometria das interações de halogênio do composto 1,2,3-tribromo-5-nitrobenzeno.

\begin{tabular}{|c|c|c|}
\hline $\mathrm{X} 1 \bullet \bullet \times 2$ & Distância $(\AA)$ & Ângulo de ligação $\left({ }^{\circ}\right)$ \\
\hline $\mathrm{Br} 2 \cdot \bullet \bullet \operatorname{Br} 11$ & $3,642(3)$ & $165,2(5)$ \\
\hline $\mathrm{Br} 1 \bullet \cdot \mathrm{Br} 1$ & $3,731(4)$ & $133,3(4)$ \\
\hline $\mathrm{Br} 2 \cdot \bullet \cdot \mathrm{Br} 13$ & $3,781(3)$ & $126,8(4)$ \\
\hline
\end{tabular}

O empacotamento molecular da 1,2,3-tribromo-5nitrobenzeno polariza os átomos de halogênio formando interações do tipo $\mathrm{Br}^{\delta+} \bullet \cdot \bullet \mathrm{Br}^{\delta-}$. Existe ainda na estrutura um contato do tipo $\mathrm{O} \bullet \bullet \mathrm{Br}$, sem maior detalhamento. A substituição do átomo de bromo pelo iodo na posição para gerou o 1,3-dibromo-2-iodo-benzeno e alterou drasticamente o empacotamento molecular formando uma rede de interações de halogênio mais complexa conforme observado na Tabela 3. A polarização observada para interação $\mathrm{Br} 1 \cdots \cdot \bullet_{112}$ foi $\mathrm{Br}^{\delta+} \bullet \bullet I^{\delta-}$, embora fosse esperada uma polarização contrária devido à baixa eletronegatividade e à alta polarizabilidade do iodo se comparada ao átomo de bromo ${ }^{23}$. 
Tabela 3. Geometria das interações de halogênio do composto 1,3-dibromo-2-iodo-5-nitrobenzeno.

\begin{tabular}{|c|c|c|}
\hline $\mathbf{X 1} \ldots \mathbf{X} \mathbf{2}$ & Distância $(\mathbf{\AA})$ & Ângulo de ligação $\mathbf{(}^{\mathbf{}} \mathbf{)}$ \\
\hline $\mathrm{Br} 1 \ldots \mathrm{I} 12$ & $3,813(2)$ & $161,2(4)$ \\
\hline $\mathrm{I} 2 \ldots \mathrm{Br} 11$ & $3,893(2)$ & $116,6(4)$ \\
\hline $\mathrm{Br} 1 \ldots \mathrm{Br} 13$ & $3,787(2)$ & $142,8(4)$ \\
\hline $\mathrm{Br} 11 \ldots \mathrm{Br} 3$ & $3,858(2)$ & $143,9(4)$ \\
\hline
\end{tabular}

Em 2016, Rajesh Gound e colaboradores ${ }^{10}$ analisaram as interações do iodo na molécula 1,3,5-triiodo-2,4,6trinitrobenzeno em co-cristais com as seguintes estruturas: 4,4-bipiridina; 4-dimetilaminopiridina; 4(etilaminometil) piridina; 4-picolina-N-oxido; piridina-N-oxido e tiouréia. Inicialmente, a determinação das interações ocorreu baseadas em padrões geométricos ${ }^{10}$. Foi concluído que o grande potencial eletrostático positivo na região do $\sigma-$ hole $^{9}$ dos átomos de iodo possibilitou interações de halogênios direcionais e muito curtas nos co-cristais estudados. Os cocristais 1,3,5-triiodo-2,4,6-trinitrobenzeno:tiourea e 1,3,5 triiodo-2,4,6-trinibrobenzeno:4-picoline-N-oxido exibiram as interações mais curtas observadas, $\mathrm{I} \cdots \mathrm{S}\left(3,085 \AA, 178^{\circ}\right)$ e I...O $\left(2,637 \AA, 174,9^{\circ}\right)$, respectivamente ${ }^{10}$. A distância I••O $\left(2,637 \AA, 174,9^{\circ}\right)$ é menor que a distância mínima observada por Gavezzoti em $2010^{17}$.

No artigo publicado por Jian-Rong Wang e colaboradores $^{24}$, foi observada a formação de 3 interações de halogênio em 3 co-cristais distintos. No co-cristal $2\left(\mathrm{C}_{27} \mathrm{H}_{48} \mathrm{O}\right) \cdot\left(\mathrm{C}_{6} \mathrm{H}_{5} \mathrm{BrO}\right)$, foi identificada a interação $\mathrm{C}-\mathrm{Br} \cdots \mathrm{O}$ $(\mathrm{dBr} . . \mathrm{O}=3,12 \AA)$ enquanto que em $\left(\mathrm{C}_{6} \mathrm{H}_{8} \mathrm{O}_{6}\right) \cdot\left(\mathrm{C}_{5} \mathrm{H}_{4} \mathrm{BrNO}\right)$ foi observada uma interação $\mathrm{C}-\mathrm{Br} \bullet \bullet \mathrm{O}$ com distância maior $(\mathrm{dBr} . . \mathrm{O}=3,29 \AA)$ e em $2\left(\mathrm{C}_{27} \mathrm{H}_{48} \mathrm{O}\right) .(\mathrm{C} 6 \mathrm{H} 5 \mathrm{IO})$, a distância entre os átomos de iodo e oxigênio C-I $\cdots O \mathrm{O}(\mathrm{dI} \ldots \mathrm{O}=3,08$ $\AA)^{24}$. As interações foram analisadas a partir de padrões geométricos.

No empacotamento molecular de quatro estruturas cristalinas para-substituídas de derivados de 2,6-dimetilbromobenzeno publicada por Angélica Navarrete Guitérrez ${ }^{25}$ e colaboradores, não foram observadas interações intermoleculares do átomo de bromo em nenhuma das estruturas cristalinas. Foram utilizados substituintes retiradores de elétrons $\left(\mathrm{CN}, \mathrm{NO}_{2}\right)$ e grupos doadores de elétrons $\left(\mathrm{NH}_{2}, \mathrm{OH}\right)^{25}$ substituídos na posição para em relação ao átomo de bromo.

Anthony $^{2}$ analisa, primeiramente, a natureza da ligação de halogênio na fase gás em interações do tipo B $\cdots X-Y$, na qual B é uma base de Lewis e X-Y é uma molécula de dihalogênio. Foi observado que as forças envolvidas na formação de uma ligação de halogênio incluem as de origem eletrostáticas, as decorrentes da transferência de cargas entre doador e aceitador e as decorrentes da dispersão, bem como sua relação com a geometria dessas interações. Também foi observado que quanto mais forte a ligação de halogênio, mais linear ela é e menor é a distância de interação $0^{2}$.

Lucas e colaboradores ${ }^{26}$ enfatizam o fato de que Ligações de Halogênio ocorrem graças às interações entre o orbital $\sigma^{*} \mathrm{C}-\mathrm{X}$ e ainda estabelecem uma relação direta entre o valor máximo da energia potencial eletrostática no $\sigma-$ hole $^{9}$ e a energia do orbital LUMO do doador da Ligação de Halogênio, ressaltando a importância da correlação eletrônica nos cálculos teóricos que envolvam Ligações de Halogênio.

Um novo potencial empírico foi introduzido, baseado em parâmetros quânticos, a fim de descrever sistemas gerais guiados por Ligações de Halogênio, com a finalidade de aprimorar a descrição dos átomos de flúor, cloro e bromo em interações de Halogênio. E, desta forma, concluíram que o conjunto de funções de bases aug-cc-pVTZ, e os funcionais B97-D3BJ, B3PYP-D3BJ e B3LYP-D3 apontaram melhores resultados para o estudo dos potencias eletrostáticos e da energia de interação ${ }^{26}$. Concluiu ainda que é possível baixar o custo computacional usando o conjunto de funções de bases $6-31+\mathrm{G}^{* *}$ e os funcionais B97-DEBJ e B3PYP-D3BJ sem perda de qualidade significativa para os cálculos teóricos. Foram testados os funcionais B97D3BJ, B97-D3, B3PYP-D3BJ, B3LYP-D3 e M062X-D3 e as funções de base aug-cc-pVTZ e 6-31+ $\mathrm{G}^{* * 26}$.

Gabriella Cavallo e colaboradores ${ }^{1}$ analisaram o estado da arte da ligação de halogênio, bem como elencaram as novas aplicações dessa classe de interações em diversas áreas e fazem ainda uma breve revisão histórica sobre o tema. Eles concluíram que nos últimos 15 anos os dados 
experimentais e teóricos obtidos contribuíram para o desenvolvimento e a compreensão da atual natureza da interação de halogênio, bem como de suas potenciais aplicações nas mais diversas áreas ${ }^{1}$. O trabalho deles mostra que os dados teóricos gerados a partir de cálculos $a b$ initio contribuem inequivocamente com o desenvolvimento do tema, bem como suas possíveis aplicações, auxiliando na compreensão dessa classe de interações em ambientes complexos ${ }^{1}$.

Dados teóricos e experimentais demostram que entre os átomos de halogênio, a tendência em formar ligações fortes segue a seguinte ordem: $\mathrm{I}>\mathrm{Br}>\mathrm{Cl}>\mathrm{F} 1$. Muitos processos envolvidos nessa classe de interações puderam ser revelados a partir das abordagens teóricas disponíveis corroboradas pelos dados experimentais. A força de interação intermolecular pode ser estimada utilizando a Teoria Quântica de Átomos em Moléculas (QTAIM) ${ }^{27,28,12}$, suportada pelo entendimento dos orbitais naturais de ligação (NBOs) $)^{13,14,15}$ e dos orbitais HOMO e LUMO. Somando a isso, as principais regiões de interação entre o aceptor e o doador podem ser identificadas através da superfície de potencial eletrostático (MEP - Molecular Electrostatic Potential)

\section{Conclusões}

A partir da revisão sistemática realizada, foi possível identificar o estado da arte em termos de Ligações de Halogênio em compostos derivados de 2,6-dibromonitrozeno. Na maioria dos casos, as interações de halogênio em compostos derivados de nitrobenzeno foram avaliadas somente por padrões geométricos. Foram poucos os casos nos quais as interações foram avaliadas com o apoio de cálculos teóricos. Foi possível concluir que os funcionais B97-D3BJ, B3PYPD3BJ e B3LYP-D3 juntamente às funções de base aug-ccpVTZ e $6-31+G^{* *}$ apontaram melhores resultados para o estudo do potencial eletrostáticos e da energia de interação, bem como a importância da correlação eletrônica nos cálculos teóricos.

Nem mesmo a teoria do QTAIM ${ }^{28,27,28}$ foi empregada para estudo dessa classe de compostos. Verifica-se, pelos artigos estudados, a necessidade de uma abordagem teórica robusta que forneça uma melhor caracterização dessa classe de interações em compostos derivados de nitrobenzeno. E uma das formas de caracterizar essas interações é pelo estudo topológico da QTAIM2 $2^{8,27,28}$.

Cálculos teóricos, como os orbitais naturais de ligação $(\mathrm{NBOs})^{15}$, juntamente aos orbitais HOMO e LUMO torna possível a determinação dos orbitais envolvidos na formação das interações, bem como a energia envolvida em cada interação. Os orbitais $(\mathrm{NBOs})^{15}$ proporcionam uma representação precisa da estrutura de Lewis (NLS) ${ }^{29}$, bem como a localização dos orbitais atômicos naturais (NAOs), a deslocalização da estrutura eletrônica e do balanço de cargas. O mapa de potencial eletrostático (MEP) fornecerá importantes contribuições para identificação da região do $\sigma-$ hole $^{9}$, facilitando a identificação de sítios aceitadores e doadores nas moléculas.

Com base nos dados pesquisados, sugerem-se estudos teóricos, utilizando a Teoria do Funcional de Densidade $(\mathrm{DFT})^{16}$ e a teoria do QTAIM para análise das interações intermoleculares em compostos para substituídos de 2,6-dibromonitrozeno com os substituintes $(\mathrm{Cl}, \mathrm{Br}$, $\mathrm{F}, \mathrm{I})$. Tendo em vista que o grupo nitro ligado ao anel benzeno pode atuar com uma base de Lewis forte, devido à alta densidade eletrônica dos oxigênios e ao seu efeito indutivo retirador de elétrons que, juntamente aos átomos de halogênio na molécula, podem provocar interações complexas no empacotamento molecular.

\section{Referências}

1. Cavallo G, Metrangolo P, Milani R, et al. The Halogen Bond. Chem Rev. 2016;116(4):2478-2601. doi:10.1021/acs.chemrev.5b00484.

2. Legon AC. The halogen bond: an interim perspective. Phys Chem Chem Phys. 2010;12(28):7736-7747. doi:10.1039/C002129F.

3. Benesi HA, Hildebrand JH. ULTRAVIOLET ABSORPTION BANDS OF IODINE IN AROMATIC HYDROCARBONS. J Am Chem Soc. 1948;70(8):2832-2833. doi:10.1021/ja01188a528.

4. Benesi HA, Hildebrand JH. A Spectrophotometric Investigation of the Interaction of Iodine with Aromatic Hydrocarbons. J Am Chem Soc. 1949;71(8):2703-2707. doi:10.1021/ja01176a030.

5. Mulliken RS. Structures of Complexes Formed by Halogen Molecules with Aromatic and with Oxygenated Solvents1. J Am Chem Soc. 1950;72(1):600-608. doi:10.1021/ja01157a151. 
6. O. Hassel; Knut O. Stromme. Crystal Structure of the Addition Compound 1,4-Dioxan-Chlorine. Acta Chem Scand - ACTA CHEM SCAND. 1959;13:1775-1780.

7. Odd Hassel. Strutural Aspects of Interatomic Charge-Transfer Bonding. Science (80- ). 1970;170(3957).

8. Dahl T, Hassel O, J. Ballhausen C, et al. Solid Adducts of Hexamethylenetetramine and Trihalogenomethanes. Crystal Structure of the 1:1 Iodoform Compound. Vol 24.; 1970. doi:10.3891/acta.chem.scand.24-0377.

9. Clark T, Hennemann M, Murray J, Politzer P. Halogen Bonding: The б-Hole. Vol 13.; 2007. doi:10.1007/s00894-006-0130-2.

10. Goud NR, Bolton O, Burgess EC, Matzger AJ. Unprecedented Size of the $\sigma$-Holes on 1,3,5-Triiodo-2,4,6-trinitrobenzene Begets Unprecedented Intermolecular Interactions. Cryst Growth Des. 2016;16(3):1765-1771. doi:10.1021/acs.cgd.6b00074.

11. Spackman MA, Jayatilaka D. Hirshfeld surface analysis. CrystEngComm. 2009;11(1):19-32. doi:10.1039/B818330A.

12. Syzgantseva OA, Tognetti V, Joubert L. On the Physical Nature of Halogen Bonds: A QTAIM Study. J Phys Chem A. 2013;117(36):8969-8980. doi:10.1021/jp4059774.

13. Pounds AJ. Valency and Bonding: A Natural Bond Orbital DonorAcceptor Perspective (Frank Weinhold and Clark Landis). J Chem Educ. 2007;84(1):43. doi:10.1021/ed084p43.

14. Weinhold F. Natural bond orbital analysis: A critical overview of relationships to alternative bonding perspectives. J Comput Chem. 2012;33(30):2363-2379. doi:10.1002/jcc.23060.

15. Foster JP, Weinhold F. Natural hybrid orbitals. J Am Chem Soc. 1980;102(24):7211-7218. doi:10.1021/ja00544a007.

16. Kohn W, Sham LJ. Self-Consistent Equations Including Exchange and Correlation Effects. Phys Rev. 1965;140(4A):A1133-A1138. doi:10.1103/PhysRev.140.A1133.

17. Gavezzotti A. Non-conventional bonding between organic molecules. The 'halogen bond' in crystalline systems. Mol Phys. 2008;106(12-13):1473-1485. doi:10.1080/00268970802060674.

18. Groom CR, Bruno IJ, Lightfoot MP, Ward SC. The Cambridge Structural Database. Acta Crystallogr Sect B Struct Sci Cryst Eng Mater. 2016;72(2):171-179. doi:10.1107/S2052520616003954.

19. Frisch MJ, Trucks GW, Schlegel HB. Gaussian 03, Revision B.04. Vol null. (null, ed.).; 2003.

20. Merz K. Substitution Effect on Crystal Packings of Iodobenzonitriles and Iodophenols. Cryst Growth Des. 2006;6(7):1615-1619. doi:10.1021/cg060067j.

21. Mossakowska I, Wójcik GM. Supramolecular assemblies of singlesubstituted nitrobenzenes in their experimental and predicted crystal structures. J Mol Struct. 2010;967(1):119-130. doi:https://doi. org/10.1016/j.molstruc.2009.12.047.

22. Hoque MM, Halim MA, Rahman MM, Hossain MI, Khan MW. Synthesis and structural insights of substituted 2-iodoacetanilides and 2-iodoanilines. J Mol Struct. 2013;1054-1055:367-374. doi:https://doi.org/10.1016/j.molstruc.2013.10.011.

23. Romero JA, Aguirre Hernández G, Bernès S. Anomalous halogen bonds in the crystal structures of 1,2,3-tri-bromo-5-nitro-benzene and 1,3-di-bromo-2-iodo-5-nitro-benzene. Acta Crystallogr Sect E, Crystallogr Commun. 2015;71(Pt 8):960-964. doi:10.1107/ S2056989015013377.
24. Wang J-R, Fan X, Ding Q, Mei X. Determination of absolute configuration using heavy atom based co-crystallization method: Halogen atom effects. J Mol Struct. 2016;1119:269-275. doi:https:// doi.org/10.1016/j.molstruc.2016.04.084.

25. Navarrete Guitérrez A, Aguirre Hernández G, Bernès S. Crystal structures of p-substituted derivatives of 2,6-di-methylbromo-benzene with $1 / 2 \leq Z^{\prime} \leq 4$. Acta Crystallogr Sect E, Crystallogr Commun. 2016;72(Pt 12):1762-1767. doi:10.1107/ S2056989016017485.

26. Santos LA, da Cunha EFF, Ramalho TC. Toward the Classical Description of Halogen Bonds: A Quantum Based Generalized Empirical Potential for Fluorine, Chlorine, and Bromine. J Phys Chem A. 2017;121(12):2442-2451. doi:10.1021/acs.jpca.6b13112.

27. Bader RFW. Atoms in Molecules. Vol null. (null, ed.).; 1990.

28. Bader RFW. Atoms in molecules. Acc Chem Res. 1985;18(1):9-15. doi:10.1021/ar00109a003.

29. Reed AE, Curtiss LA, Weinhold F. Intermolecular interactions from a natural bond orbital, donor-acceptor viewpoint. Chem Rev. 1988;88(6):899-926. doi:10.1021/cr00088a005.

\section{Ricardo R. Ternavisk ${ }^{1 *}, 3$, Gerardo A. Hernández², Jean M. F. Custódio ${ }^{4}$, Ademir J. Camargo' \& Hamilton B. Napolitano ${ }^{1,5}$}

${ }^{1}$ Universidade Estadual de Goiás, 75001-970, Anápolis, GO, Brasil.

${ }^{2}$ Instituto Tecnológico de Tijuana, Apdo. Postal 1166, 22510 Tijuana, B.C., México.

${ }^{3}$ Universidade Paulista, 74845-090, Goiânia, GO, Brasil.

${ }^{4}$ Universidade Federal de Goiás, 74690-900, Goiânia, GO, Brasil.

${ }^{5}$ Centro Universitário de Anápolis, 75083-515, Anápolis, GO, Brasil.

*E-mail: ternavisk130@gmail.com 